\title{
Pharmacokinetics of oral and intravenous melatonin in healthy volunteers
}

\author{
Lars P. H. Andersen 1*, Mads U. Werner ${ }^{2}$, Mette M. Rosenkilde ${ }^{3}$, Nathja G. Harpsøe ${ }^{1}$, Hanne Fuglsang ${ }^{1}$, \\ Jacob Rosenberg ${ }^{1}$ and Ismail Gögenur ${ }^{4}$
}

\begin{abstract}
Background: The aim was to investigate the pharmacokinetics of oral and iv melatonin in healthy volunteers.

Methods: The study was performed as a cohort crossover study. The volunteers received either $10 \mathrm{mg}$ oral melatonin or $10 \mathrm{mg}$ intravenous melatonin on two separate study days. Blood samples were collected at different time points following oral administration and short iv infusion, respectively. Plasma melatonin concentrations were determined by RIA technique. Pharmacokinetic analyses were performed by "the method of residuals" and compartmental analysis. The pharmacokinetic variables: $k_{a}, t_{1 / 2}$ absorption, $t_{\max }, C_{\max }, t_{1 / 2}$ elimination, $A U C_{0-\infty}$, and bioavailability were determined for oral melatonin. $C_{\max }, t_{1 / 2}$ elimination, $V_{d}, C L$ and $A U C_{0-\infty}$ were determined for intravenous melatonin.

Results: Twelve male volunteers completed the study. Baseline melatonin plasma levels did not differ significantly between the study days $(P=0.067)$. Mean (SD) $t_{1 / 2}$ absorption of oral melatonin was 6.0 (3.1) min. Mean $t_{\max }$ was 40.8 (17.8) min with a median (IQR) $C_{\max }$ of 3550.5 (2500.5-8057.5) $\mathrm{pg} \mathrm{ml}^{-1}$. Mean $t_{1 / 2}$ elimination was 53.7 (7.0) min. Median absolute bioavailability was 2.5 (1.7-4.7) \%. Median $C_{\max }$ after short iv infusion of melatonin was 389,875.0 $(174,775.0-440,362.5) \mathrm{pg} \mathrm{ml}^{-1}$. Mean $t_{1 / 2}$ elimination was 39.4 (3.6) min, mean $V_{\mathrm{d}} 1.2(0.6) \mathrm{I} \mathrm{kg}^{-1}$ and mean $C L 0.0218$ (0.0102) I $\mathrm{min}^{-1} \mathrm{~kg}^{-1}$.
\end{abstract}

Conclusions: This cohort crossover study estimated pharmacokinetics of oral and iv melatonin, respectively in healthy volunteers. Bioavailability of oral melatonin was only $3 \%$.

Trial registration: Eudra-CT number: 2013-000205-23 (initial registration 27.03.2013).

Clinicaltrials.gov Identifier: NCT01923974 (initial registration 08.08.2013).

Keywords: Bioavailability, Intravenous, Melatonin, Oral, Pharmacokinetic

\section{Background}

Exogenous melatonin is being increasingly employed as treatment for various medical and surgical diseases $[1,2]$. Furthermore, a recent study, administering intravenous (iv) melatonin has documented reduced cardiac morbidity and markers of myocardial ischemia following elective abdominal aortic aneurism repair [3]. Despite its widespread clinical use, the pharmacokinetic properties of exogenous melatonin still need to be established further [4]. A limited number of experimental studies in healthy volunteers have performed direct comparisons of the pharmacokinetics of

\footnotetext{
* Correspondence: Iphandersen@gmail.com

${ }^{1}$ Department of Surgery D, Herlev Hospital, University of Copenhagen, Herlev DK-2730, Denmark

Full list of author information is available at the end of the article
}

oral and iv melatonin $[5,6]$. The studies differed in number of investigated subjects, dosages, methods and pharmacokinetic analyses $[5,6]$. Accordingly, the pharmacokinetic variables varied extensively between the studies $[5,6]$. In order to achieve an optimized clinical efficacy of melatonin, further investigation of the pharmacokinetics is clearly needed.

The aim of the study was to investigate the pharmacokinetics of oral and iv melatonin in a cohort of healthy volunteers.

\section{Methods}

Approvals were obtained from the Capital Region's Committee on Health Research Ethics (Protocol number: H-4-2013-013), the Danish Health and Medicines 
Authority (Eudra-CT number: 2013-000205-23, Clinicaltrials.gov Identifier: NCT01923974) and the Danish Data Protection Agency (Journal number: HEH-2013-008, nr: 02095) prior to inclusion of volunteers. Informed writtenand verbal consent were provided by all volunteers. The blood samples relating to iv melatonin were obtained from a primary trial investigating analgesic and anti-hyperalgesic effects of melatonin (Eudra-CT number: 2013-000205-23, Clinicaltrials.gov Identifier: NCT01923974). Data presented in the present paper have not been published previously.

The study was performed as a cohort crossover study. The volunteers received either $10 \mathrm{mg}$ of oral melatonin or $10 \mathrm{mg}$ of iv melatonin on two separate study days. Each study session was performed from 08:00 a.m. to 04:00 p.m. The study days were separated by 3 to 9 months. Inclusion criteria were age 20-40 years and male gender. Volunteers were excluded, if they did not understand written- or spoken Danish, suffered from serious physical or mental illness, were diagnosed with a sleep disorder, worked night shifts, received daily analgesics, had participated in other clinical trials within one month from study inclusion or suffered from skin abnormalities on the lower extremities (due to the test paradigm applied in the primary trial).

Oral melatonin consisted of one gelatine capsule containing $10 \mathrm{mg}$ melatonin. Volunteers were allowed $5 \mathrm{cl}$ of tap water to facilitate oral intake. Volunteers were instructed to adhere to preoperative fasting guidelines before the study session with oral melatonin (liquids: minimum fasting period $=2 \mathrm{~h}$; meals: minimum fasting period $=6 \mathrm{~h}$ ) [7]. Volunteers were encouraged to drink and eat $2 \mathrm{~h}$ following oral melatonin administration. Intravenous melatonin consisted of a $25 \mathrm{ml}$ ethanol/saline solution ( $2 \mathrm{ml} 99.9 \%$ ethanol $/ 23 \mathrm{ml} 0.9 \%$ saline) containing $10 \mathrm{mg}$ of melatonin (Helsinn Chemicals SA, Biasca, Switzerland). Intravenous melatonin was administered as short iv infusion in the left antecubital vein (2.5 ml min ${ }^{-1} ; 10$ min duration).

Blood samples were collected from a peripheral venous catheter inserted in the right antecubital vein at specified time points during each study session. The time points differed between oral and iv melatonin. Oral melatonin: at baseline (before medication $=$ endogenous melatonin production), and $0,10,20,30,40,50,60,70$, $80,90,100,110,120,180,240,300,360$ and $420 \mathrm{~min}$ after oral administration. Intravenous melatonin: at baseline, and $0,60,120,180,240,300,360$ and $420 \mathrm{~min}$ after short iv infusion. Before each blood sample was collected, a $3 \mathrm{ml}$ volume of blood (residual volume) was drawn from the peripheral venous catheter and discarded. Fractionation of blood samples was performed at 5000 r.p.m. for $5 \mathrm{~min}$, and plasma samples were stored at $-80^{\circ} \mathrm{C}$. Quantitative determination of plasma melatonin concentrations was performed by radioimmunoassay (RIA)-technique (Melatonin Direct RIA, DIAsource, Louvaine-La-Neuve, Belgium). Precision of the RIA kit: intra-assay coefficient of variation $(\mathrm{CV})=9.8-13.4 \%$, inter-assay $\mathrm{CV}=8.0-13.3 \%$. The limit of detection was $2.3 \mathrm{pg} \mathrm{ml}^{-1}$. Linearity of the kit ranged between $8.5-$ $529.0 \mathrm{pg} \mathrm{ml}^{-1}$. If plasma concentrations exceeded detection range of the kit, plasma samples were diluted according to manufacturer's guidelines. All plasma samples were analysed in duplicate, and the mean value was reported.

\section{Statistical and pharmacokinetic analyses}

Normality of data was assessed by visual inspection of residual plots and histograms. Parametric or non-parametric tests were applied according to the distribution of data. Correspondingly, data are presented as mean (SD) or median (IQR), unless stated otherwise. A $P$-value $<0.05$ is considered statistically significant. Data were analysed using IBM SPSS Statistics for Windows version 22.0 (IBM Corp., Armonk, NY, USA) and Graph Pad Prism version 6.0 (Graph Pad Software Inc., La Jolla, CA, USA).

The baseline melatonin plasma concentrations of each study day were compared using a paired sample T-test. Baseline levels were not subtracted from post-treatment (oral or iv) levels.

Pharmacokinetic analyses of oral and iv melatonin were performed separately.

\section{Oral melatonin}

Time to maximal concentrations $\left(t_{\max }\right)$ and maximal plasma concentrations $\left(C_{\max }\right)$ were assessed directly at the relevant time points. The pharmacokinetic variables: absorption constant $\left(k_{\mathrm{a}}\right)$, absorption half-life $\left(t_{1 / 2}\right.$ absorption $)$, elimination rate constant $\left(k_{\mathrm{e}}\right)$ and elimination half-life $\left(t_{1 / 2}\right.$ elimination $)$ were estimated by "the method of residuals" [8]. Areas-under-the-curve (AUC) of plasma concentrations were calculated by applying the trapezoidal rule [9]. $A U C_{0-\infty}$ was estimated as $A U C_{0-420 \mathrm{~min}}+\left(C_{420 \mathrm{~min}} /\right.$ $\left.k_{\mathrm{e}}\right)$. Bioavailability was calculated as $\left(\mathrm{AUC}_{0-\infty}\right.$ oral $/$ $\mathrm{AUC}_{0-\infty}$ IV) $\mathrm{x} 100$.

\section{Intravenous melatonin}

$C_{\max }$ was assessed directly at the time point, $0 \mathrm{~min}$ after short iv infusion. Pharmacokinetic variables were calculated by compartmental analysis [10]. The pharmacokinetic variables: $t_{1 / 2}$ elimination, volume of distribution $\left(V_{\mathrm{d}}\right)$ and clearance $(C L)$ were estimated from individual linear regression lines of log-transformed (natural logarithm) plasma concentrations. Following standard equations were applied: $t_{1 / 2}$ elimination $=\ln (2) / k_{\mathrm{e}}, V_{\mathrm{d}}=$ dose $/ C_{0}$ min, $C L=k_{\mathrm{e}} \times V_{\mathrm{d}}$. "Goodness of fit" of the individual linear regression lines was assessed by the coefficient of determination, $\mathrm{R}^{2} . A U C_{0-\infty}$ IV was estimated, as described above. 


\section{Results}

Twelve male volunteers were included and completed the study. Mean age and body mass index (BMI) were 27.1 (5.2) years and $23.2(2.7) \mathrm{kg} \mathrm{m}^{-2}$, respectively. Baseline melatonin plasma concentrations did not differ significantly between the study days (before oral melatonin = 27.3 (13.5) $\mathrm{pg} \mathrm{ml}^{-1}$; before intravenous melatonin $=18.3$ (12.3) $\left.\mathrm{pg} \mathrm{ml}^{-1}\right)(P=0.067)$.

The pharmacokinetic variables of oral and iv melatonin are presented in Tables 1 and 2.

\section{Oral melatonin}

Oral melatonin demonstrated first-order absorption and elimination kinetics. Mean $k_{\mathrm{a}}$ was $0.2(0.1) \mathrm{min}^{-1}$, and mean $t_{1 / 2}$ absorption of oral melatonin was $6.0(3.1) \mathrm{min}$ (Fig. 1). Mean $t_{\max }$ was 40.8 (17.8) min with a median (IQR) $C_{\max }$ of $3550.5(2500.5-8057.5) \mathrm{pg} \mathrm{ml}^{-1}$. Mean $t_{1 / 2}$ elimination was $53.7(7.0) \mathrm{min}, A U C_{0-\infty}$ oral $281,538.3$ $(232,696.1-546,285.4) \mathrm{pg} \mathrm{ml}^{-1} \mathrm{~min}$ and median absolute bioavailability was $2.5(1.7-4.7) \%$.

\section{Intravenous melatonin}

The pharmacokinetic profiles of iv melatonin demonstrated first-order elimination kinetics (Fig. 2). Median $C_{\max }$ after iv bolus injection of $10 \mathrm{mg}$ melatonin was $389,875.0(174,775.0-440,362.5) \mathrm{pg} \mathrm{ml}^{-1}$. Mean $t_{1 / 2}$ elimination was 39.4 (3.6) min, mean $V_{\mathrm{d}} 1.2(0.6) \mathrm{l} \mathrm{kg}^{-1}$ and mean $C L$ $0.0218(0.0102) 1 \mathrm{~min}^{-1} \mathrm{~kg}^{-1}$. Median $\mathrm{R}^{2}$ was $0.96(0.93-$ 0.97). Median $A U C_{0-\infty}$ IV vas $14,179,767.6(7,063,347.4-$ $18,964,804.0) \mathrm{pg} \mathrm{ml}^{-1} \mathrm{~min}$.

\section{Discussion}

This cohort crossover study demonstrated a $t_{\max }$ of 41 min following oral administration. $C_{\max }$ and $A U C$ varied extensively between volunteers in both administration routes. Elimination half-lives were $54 \mathrm{~min}$ and 39 min, respectively. Bioavailability of oral melatonin was only $3 \%$, but demonstrated substantial inter-individual differences.

\section{Oral melatonin}

Oral melatonin was absorbed by first-order kinetics, which has previously been demonstrated in doses up to $80 \mathrm{mg}$ [8]. The short $t_{1 / 2}$ absorption of $6 \mathrm{~min}$, corroborate studies, applying similar oral drug formulations [8]. Accordingly, our $t_{\max }$ value of $41 \mathrm{~min}$ is in agreement with other studies, documenting values ranging from 30 to
60 min $[6,11]$. Oral administration of exogenous melatonin, approximately $45 \mathrm{~min}$ before intended onset of clinical effects therefore seems reasonable, assuming that clinical efficacy coincides with $t_{\max }$ values [12]. Oral administration was associated with extremely variable $C_{\max }$ and $A U C_{0-\infty}$ oral values, which has been described previously [5]. The inter-individual variations are apparently caused by differences in absorption, distribution, metabolism or excretion of the drug, but the exact causes and clinical implications remain unestablished so far [5]. Previous studies demonstrate $t_{1 / 2}$ elimination values ranging from 46 to $65 \mathrm{~min}$ in oral doses from 0.5 to $6 \mathrm{mg}[5,6,11]$, which correlates with our findings of $54 \mathrm{~min}$. Our data demonstrated a very low absolute bioavailability of $3 \%$, albeit with a substantial inter-individual variability. Previous experimental studies have documented higher values ranging between 9 and $33 \%$, although with comparable interindividual variability $[5,6,12]$. It is well established in both animal- and human studies that the low bioavailability results from an extensive hepatic first pass metabolism [5]. Similarly, it is also clear that these findings may mandate future dose regulations between different administration routes. However, a general lack of experimental- and clinical studies correlating melatonin plasma concentration levels and clinical effects still remains, and further knowledge is needed, preferably by in-depth pharmacokineticpharmacodynamic modelling.

\section{Intravenous melatonin}

Previous studies investigating iv administration of melatonin have also demonstrated first-order eliminations kinetics [10], as observed in our study. As with oral melatonin, iv administrations displayed extensive variations in $C_{\max }$ and $A U C_{0-\infty}$ IV values, which is in accordance with previous studies [6]. Other studies also documented $t_{1 / 2}$ elimination values ranging between 28 and $60 \mathrm{~min}$ in iv doses from $0.005 \mathrm{mg}$ to $2 \mathrm{mg}[6,10,13]$, which corresponds to the $39 \mathrm{~min}$, demonstrated in the present study. Several studies confirm that elimination rates of iv melatonin (and oral melatonin) are not related to the administered dose. Similarly, previous studies document $C L$ values of $0.013 \mathrm{l} \mathrm{min}^{-1} \mathrm{~kg}^{-1}$ (weight-corrected) [13] and $0.027 \mathrm{l} \mathrm{min}^{-1} \mathrm{~kg}^{-1}$ [10], which correspond well to our findings of $0.0221 \mathrm{~min}^{-1} \mathrm{~kg}^{-1}$. Cavallo and colleagues also documented a $V_{\mathrm{D}}$ of $1.8 \mathrm{l} \mathrm{kg}^{-1}$ [10], which is comparable with a value of $1.21 \mathrm{~kg}^{-1}$, demonstrated in our study.

Table 1 Pharmacokinetic variables of $10 \mathrm{mg}$ of oral melatonin

\begin{tabular}{lllll}
\hline$t_{1 / 2 \text { absorption } \min }$ & $t_{\max } \min$ & $C_{\max } \mathrm{pg} \mathrm{mL}^{-1}$ & $t_{1 / 2 \text { elimination } \min }$ & AUC $_{0-\infty}$ oral $\mathrm{pg} \mathrm{m}{ }^{-1} \mathrm{~min}$ \\
\hline $6.0(3.1)$ & $40.8(17.8)$ & $3550.5(2500.5-8057.5)$ & $53.7(7.0)$ & $281,538.3(232,696.1-546,285.4)$
\end{tabular}

Absorption half-life, time to maximal concentration and elimination half-life data are presented as mean (SD). Maximal concentration, area-under-the-curve and bioavailability data are presented as median (IQR)

Absorption half-life, $t_{1 / 2}$ absorption; time to maximal concentration, $t_{\text {maxi }}$ maximal plasma concentration, $C_{\text {maxi }}$ elimination half-life, $t_{1 / 2}$ elimination; area-under-the-curve, AUC; bioavailability, $f$ 
Table 2 Pharmacokinetic variables of $10 \mathrm{mg}$ of iv melatonin

\begin{tabular}{llllll}
\hline$C_{\max } \mathrm{pg} \mathrm{ml}^{-1}$ & $t_{1 / 2 \text { elimination } \min }$ & $V_{\mathrm{d}} \mathrm{l} \mathrm{kg}^{-1}$ & $\mathrm{CL} / \mathrm{min}^{-1} \mathrm{~kg}^{-1}$ & $\mathrm{R}^{2}$ & AUC $_{0-\infty} \mathrm{IV} \mathrm{Pg} \mathrm{ml}^{-1} \mathrm{~min}$ \\
\hline $389,875.0(174,775.0-440,362.5)$ & $39.4(3.6)$ & $1.2(0.6)$ & $0.0218(0.0102)$ & $0.96(0.93-0.97)$ & $14,179,767.6(7,063,347.4-18,964,804.0)$
\end{tabular}

Maximal concentration, coefficient of determination and area-under-the-curve data are presented as median (IQR). Elimination half-life, volume of distribution and clearance data are presented as mean (SD)

Maximal plasma concentration, $C_{\max }$ elimination half-life, $t_{1 / 2}$ elimination; volume of distribution, $V_{\mathrm{d}}$; clearance, $C L$; coefficient of determination, $R^{2}$; area-under-the-curve, AUC

\section{Strengths}

Our study is the first to perform direct comparisons of pharmacokinetics of oral and iv melatonin in doses routinely administered perioperatively (approximately $10 \mathrm{mg}$ ) [2]. The study was performed as a crossover study to reduce the effect of the inter-individual variability on pharmacokinetic data. Our experimental setup included multiple blood samples for a detailed description of both absorption and elimination phases in both administration routes. Our study also included standard pharmacokinetic methods, such as "the method of residuals" and compartmental analysis $[8,10]$. In addition, we chose to include the coefficient of determination $\left(\mathrm{R}^{2}\right)$ to document the "goodness of fit" of the individual linear regression lines in the compartmental analysis. Our data demonstrated a $R^{2}$ value of 0.96 , indicating a high degree of "fit" of the firstorder pharmacokinetic model, and, hence, a considerable accuracy of the derived pharmacokinetic variables.

\section{Limitations}

First, this study only included healthy male volunteers in an experimental setup. Hence, a potential gender difference in pharmacokinetic variables may exist. Furthermore, previous experimental studies indicate that the pharmacokinetics of melatonin is affected by age [10] and external factors, such as caffeine intake [14] cigarette smoking [15] and the use of oral contraceptives [16]. Also, a low number of clinical studies have demonstrated altered pharmacokinetic variables of melatonin [17-19] in e.g. critically ill patients $[17,18]$. Interestingly, most other patient groups, e.g. surgical patients, still remain to be

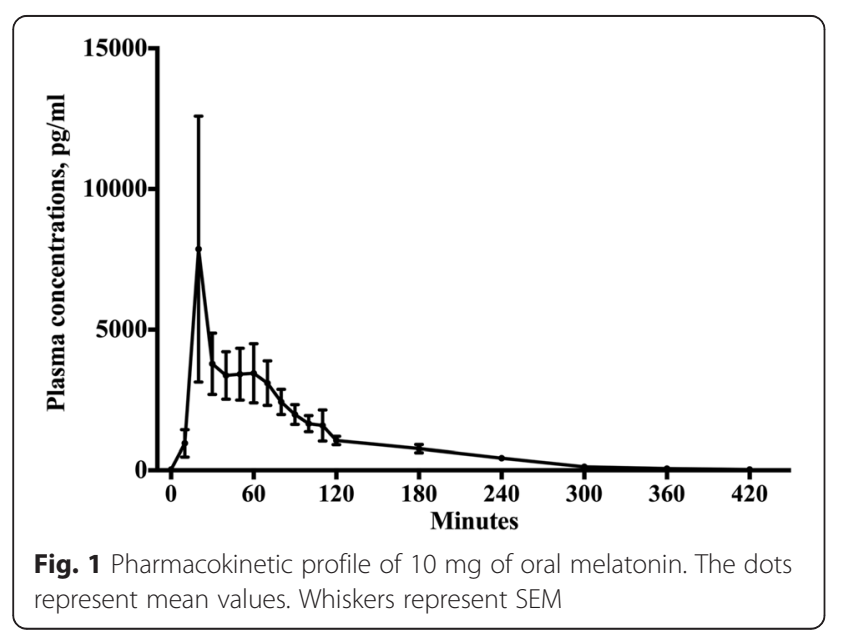

investigated. Comorbidity and drug interactions may change the pharmacokinetics of melatonin, potentially altering clinical efficacy of the drug [20].

Second, oral and iv study sessions were separated by 3 to 9 months for each volunteer. These time periods may theoretically have affected the comparability of individual pharmacokinetic variables, despite the crossover design. It, however, seems unlikely, as all volunteers were healthy young males in stable physical conditions.

Third, the very low bioavailability of oral melatonin documented in our study may indicate a deficient absorption of the drug in our setup. The volunteers were allowed $5 \mathrm{cl}$ of tap water to facilitate intake of oral melatonin. Hence, it can be discussed, if the restricted liquid volume, despite saliva and gastric/intestinal fluid secretions was sufficient to dissolve and present the ingested melatonin to the small intestine, where absorption is mainly achieved. We, however, chose this amount of water to standardize the experimental conditions and to imitate a clinical premedication scenario $[2,7]$. Also, we administered an easily absorbable gelatine capsule in order to optimize dissolution of the drug. Finally, comparable $t_{\max }$ values between the volunteers were demonstrated, suggesting that an impeded absorption is rather unlikely.

\section{Conclusions}

This crossover cohort study investigated the pharmacokinetics of oral and intravenous melatonin in healthy male volunteers. Oral melatonin was rapidly absorbed, and $\mathrm{T}_{\max }$ was achieved after $41 \mathrm{~min} . C_{\max }$ and $A U C$

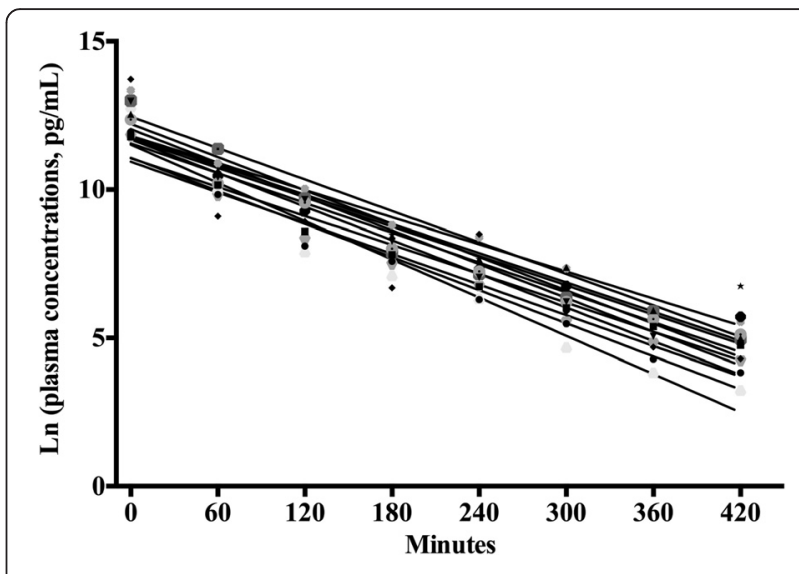

Fig. 2 Individual pharmacokinetic profiles of $10 \mathrm{mg}$ of iv melatonin 
varied extensively between volunteers. Elimination halflives following oral and intravenous melatonin administration was $54 \mathrm{~min}$ and $39 \mathrm{~min}$, respectively. The bioavailability of oral melatonin was only $3 \%$, but a considerable variability between the volunteers was noted.

\section{Competing interests}

The authors declare that they have no competing interests.

\section{Authors' contributions}

LPHA participated in the design of the study, collected data, performed dataanalysis, and drafted manuscript. MUW, MMR, JR and IG participated in the design of the study, and helped to draft the manuscript. NHG and HF collected data, and helped to draft the manuscript. All authors have read and approved the final manuscript.

\section{Author details}

'Department of Surgery D, Herlev Hospital, University of Copenhagen, Herlev DK-2730, Denmark. ${ }^{2}$ Multidisciplinary Pain Center 7612, Neuroscience Center, Rigshospitalet, Copenhagen DK-2100, Denmark. ${ }^{3}$ Department of Neuroscience and Pharmacology, University of Copenhagen, Copenhagen DK-2100, Denmark. ${ }^{4}$ Department of Surgery, Roskilde and Køge Hospital, University of Copenhagen, Roskilde DK-4000, Denmark.

Received: 11 October 2015 Accepted: 12 February 2016

Published online: 19 February 2016

\section{References}

1. Brzezinski A. Melatonin in humans. N Engl J Med. 1997;336:186-95.

2. Andersen LP, Werner MU, Rosenberg J, Gögenur I. A systematic review of peri-operative melatonin. Anaesthesia. 2014;69:1163-71.

3. Gögenur I, Kücükakin B, Panduro Jensen L, Reiter RJ, Rosenberg J. Melatonin reduces cardiac morbidity and markers of myocardial ischemia after elective abdominal aortic aneurism repair: a randomized, placebo-controlled, clinical trial. J Pineal Res. 2014;57:10-5.

4. Harpsøe NG, Andersen LP, Gögenur I, Rosenberg J. Clinical pharmacokinetics of melatonin: a systematic review. Eur J Clin Pharmacol. 2015;71:901-9.

5. Di WL, Kadva A, Johnston A, Silman R. Variable bioavailability of ora melatonin. N Engl J Med. 1997;336:1028-9.

6. DeMuro RL, Nafziger AN, Blask DE, Menhinick AM, Bertino Jr JS. The absolute bioavailability of oral melatonin. J Clin Pharmacol. 2000:40:781-4

7. American Society of Anesthesiologists Committee. Practice guidelines for preoperative fasting and the use of pharmacologic agents to reduce the risk of pulmonary aspiration: application to healthy patients undergoing elective procedures: an updated report by the American Society of Anesthesiologists Committee on Standards and Practice Parameters. Anesthesiology. 2011:114:495-511.

8. Waldhauser F, Waldhauser M, Lieberman HR, Deng MH, Lynch HJ, Wurtman RJ. Bioavailability of oral melatonin in humans. Neuroendocrinology. 1984:39:307-13.

9. Matthews JN, Altman DG, Campbell MJ, Royston P. Analysis of serial measurements in medical research. BMJ. 1990;300:230-5.

10. Cavallo A, Ritschel WA. Pharmacokinetics of melatonin in human sexual maturation. J Clin Endocrinol Metab. 1996:81:1882-6.

11. Markantonis SL, Tsakalozou E, Paraskeva A, Staikou C, Fassoulaki A. Melatonin pharmacokinetics in premenopausal and postmenopausal healthy female volunteers. J Clin Pharmacol. 2008;48:240-5.

12. Fourtillan JB, Brisson AM, Gobin P, Ingrand I, Decourt JP, Girault J. Bioavailability of melatonin in humans after day-time administration of $D(7)$ melatonin. Biopharm Drug Dispos. 2000;21:15-22.

13. Mallo C, Zaĭdan R, Galy G, Vermeulen E, Brun J, Chazot G, et al. Pharmacokinetics of melatonin in man after intravenous infusion and bolus injection. Eur J Clin Pharmacol. 1990;38:297-301.

14. Härtter S, Nordmark A, Rose DM, Bertilsson L, Tybring G, Laine K. Effects of caffeine intake on the pharmacokinetics of melatonin, a probe drug for CYP1A2 activity. Br J Clin Pharmacol. 2003;56:679-82.

15. Ursing C, von Bahr C, Brismar K, Röjdmark S. Influence of cigarette smoking on melatonin levels in man. Eur J Clin Pharmacol. 2005;61:197-201.
16. Hilli J, Korhonen T, Turpeinen M, Hokkanen J, Mattila S, Laine K. The effect of oral contraceptives on the pharmacokinetics of melatonin in healthy subjects with CYP1A2g.-163C > A polymorphism. J Clin Pharmacol. 2008;48:986-94.

17. Bourne RS, Mills GH, Minelli C. Melatonin therapy to improve nocturnal sleep in critically ill patients: encouraging results from a small randomised controlled trial. Crit Care. 2008;12:R52.

18. Mistraletti G, Sabbatini G, Taverna M, Figini MA, Umbrello M, Magni P, et al. Pharmacokinetics of orally administered melatonin in critically ill patients. J Pineal Res. 2010;48:142-7.

19. Gooneratne NS, Edwards AY, Zhou C, Cuellar N, Grandner MA, Barrett JS. Melatonin pharmacokinetics following two different oral surge-sustained release doses in older adults. J Pineal Res. 2012:52:437-45.

20. Federico S, Carrano R, Capone D, Gentile A, Palmiero G, Basile V. Pharmacokinetic interaction between levofloxacin and ciclosporin or tacrolimus in kidney transplant recipients: ciclosporin, tacrolimus and levofloxacin in renal transplantation. Clin Pharmacokinet. 2006:45:169-75.

\section{Submit your next manuscript to BioMed Central and we will help you at every step:}

- We accept pre-submission inquiries

- Our selector tool helps you to find the most relevant journal

- We provide round the clock customer support

- Convenient online submission

- Thorough peer review

- Inclusion in PubMed and all major indexing services

- Maximum visibility for your research

Submit your manuscript at www.biomedcentral.com/submit 